\title{
The N17 domain mitigates nuclear toxicity in a novel zebrafish Huntington's disease model
}

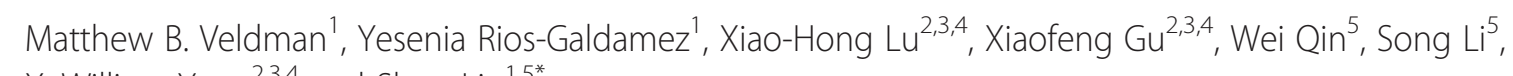
X. William Yang ${ }^{2,3,4}$ and Shuo Lin ${ }^{1,5^{*}}$

\begin{abstract}
Background: Although the genetic cause for Huntington's disease (HD) has been known for over 20 years, the mechanisms that cause the neurotoxicity and behavioral symptoms of this disease are not well understood. One hypothesis is that $\mathrm{N}$-terminal fragments of the $\mathrm{HTT}$ protein are the causative agents in HD and that peptide sequences adjacent to the poly-glutamine $(\mathrm{Q})$ repeats modify its toxicity. Here we test the function of the N-terminal 17 amino acids (N17) in the context of the exon 1 fragment of HTT in a novel, inducible zebrafish model of HD.

Results: Deletion of $\mathrm{N} 17$ coupled with $97 \mathrm{Q}$ expansion (mHTT- $\Delta \mathrm{N} 17-\mathrm{exon} 1$ ) resulted in a robust, rapidly progressing movement deficit, while fish with intact $\mathrm{N} 17$ and $97 \mathrm{Q}$ expansion (mHTT-exon1) have more delayed-onset movement deficits with slower progression. The level of $\mathrm{mHT}-\Delta \mathrm{N} 17$-exon1 protein was significantly higher than $\mathrm{mHTT}$-exon1, although the mRNA level of each transgene was marginally different, suggesting that N17 may regulate HTT protein stability in vivo. In addition, cell lineage specific induction of the $\mathrm{mHTT}-\Delta \mathrm{N} 17$-exon1 transgene in neurons was sufficient to recapitulate the consequences of ubiquitous transgene expression. Within neurons, accelerated nuclear accumulation of the toxic HTT fragment was observed in $\mathrm{mHT}-\Delta \mathrm{N} 17$-exon1 fish, demonstrating that $\mathrm{N} 17$ also plays an important role in sub-cellular localization in vivo.

Conclusions: We have developed a novel, inducible zebrafish model of HD. These animals exhibit a progressive movement deficit reminiscent of that seen in other animal models and human patients. Deletion of the N17 terminal amino acids of the huntingtin fragment results in an accelerated HD-like phenotype that may be due to enhanced protein stability and nuclear accumulation of HTT. These transgenic lines will provide a valuable new tool to study mechanisms of HD at the behavioral, cellular, and molecular levels. Future experiments will be focused on identifying genetic modifiers, mechanisms and therapeutics that alleviate polyQ aggregation in the nucleus of neurons.
\end{abstract}

Keywords: Cre inducible, Huntingtin, Huntington's disease, Polyglutamine, Zebrafish

\section{Background}

Huntington's disease (HD) is an incurable, autosomal dominant neurodegenerative disease. Patients with HD display progressive symptoms including psychiatric, cognitive, and motor dysfunction, and the disease onset

\footnotetext{
* Correspondence: shuolin@ucla.edu

'Department of Molecular, Cell and Developmental Biology, University of California-Los Angeles, 621 Charles E. Young Drive South, Los Angeles, CA 90095, USA

${ }^{5}$ Laboratory of Chemical Genomics, School of Chemical Biology and Biotechnology, Peking University Shenzhen Graduate School, Shenzhen 518055, China

Full list of author information is available at the end of the article
}

is often defined by the onset of motor symptoms [1-3]. Initially, patients present with excessive movements of the extremities and face, progressing to larger movements described as chorea. Further disease progression results in the opposite effect with patients suffering from rigidity, akinesia, bradykinesia, and gait abnormalities. The disease is invariably lethal $10-20$ years after onset. There is no cure for HD and treatments for the symptoms are of limited effectiveness.

HD is part of a broader class of neurological disorders linked to polyglutamine (polyQ) repeat expansions within several disease proteins [4]. All of these disorders 
are progressive, degenerative diseases with age of onset inversely correlated with the number of polyQ repeats within the protein. Although the genes involved in polyQ diseases are, for the most part, broadly expressed, each disease has a well-defined pattern of neurodegeneration affecting selective populations of neurons. In HD, the medium spiny neurons (MSNs) of the striatum are thought to be most susceptible to degeneration and cortical pyramidal neurons are also affected [5]. The precise mechanisms underlying such selective neuronal vulnerability in HD remain incompletely understood. One hypothesis is that amino acid sequences flanking the polyQ repeat dictate the cell-type specific toxicity.

$\mathrm{HD}$ is caused by an expanded polyQ within the Nterminus of the huntingtin protein (HTT) [6]. HTT is a large, 3144 amino acids, protein that is ubiquitously expressed, evolutionarily conserved, and has been suggested to function as a scaffolding protein for many cellular processes. Knockout of this gene is early embryonic lethal in mice suggesting an essential developmental function [7-9], however the specific mechanisms involved are not well understood. Histologically, a hallmark of $\mathrm{HD}$ is large intracellular polyQ protein aggregates [10]. These aggregates can be localized to several sub-cellular regions including cytoplasmic, perinuclear, and intra-nuclear [11, 12]. Although originally thought to be disease causative, polyQ aggregates have been suggested to be neuroprotective [13] and their role in the disease is still unresolved. One hypothesis is that localization of the aggregate defines its toxicity. Experimental targeting of HTT-polyQ to different subcellular compartments suggests that the nucleus is especially sensitive to HTT-polyQ expression $[14,15]$. The normal subcellular distribution of HTT is mediated by proteinprotein interactions to regions outside the polyQ [16]. Most notably, the N-terminal 17 amino acids of HTT (N17) have been suggested to regulate protein localization and stability [17]. This region of the protein is evolutionarily conserved [18]. It functions as both a nuclear export signal and cytoplasmic membrane association domain [19, 20]. Additionally, its amphipathic alpha-helical structure $[21,22]$ promotes oligomer formation and accelerates HTT-polyQ fragment aggregation in vitro [23, 24]. Post-translational modifications of N17 are prevalent and diverse [25]. Phosphorylation of serines 13 and 16 has been demonstrated to reduce aggregation and toxicity in vitro [26] and in vivo [27]. In vivo, BAC transgenic mice expressing human mHTT-97Q lacking the N17 domain, a deletion of 2-16 amino acid residues in otherwise fulllength mHTT context (BACHD- $\triangle \mathrm{N} 17$ ), results in greatly accelerated motor deficits including adult-onset movement disorder, striatal neurodegeneration, and exclusive nuclear mHTT aggregation formation [28]. The phenotypes of BACHD- $\triangle \mathrm{N} 17$ mice is dramatically accelerated and more closely resemble clinically-manifest HD than the BACHD mouse model expressing intact full-length mHTT of the same polyQ length [28, 29]. Therefore N17 acts as a modulator of mHTT aggregation, subcellular localization, and neurotoxicity.

Since the treatments available for HD are quite limited, it is important to have preclinical models of the disease that can be used to study mechanism and test candidate therapeutics. Mouse models are the most popular and have been developed over the last 18 years using conventional transgenic, YAC and $\mathrm{BAC}$ transgenic, and gene knock-in technologies to introduce polyQ into the genome [30]. Some models express the entire protein while others only use exon 1 which encodes the pathological polyQ expansion. These mice develop behavioral abnormalities and brain pathologies that recapitulate HD to varying degrees. Recent data suggests that proteolytic fragments containing exon 1 [31] or aberrantly spliced transcripts of exon 1 [32] are toxic and exist in patients. mHTT exon 1 based transgenic mice [33] have already been demonstrated to exhibit behavioral and pathological similarities to human disease, mHTT exon 1 based models are likely to be relevant to aspects of the disease. However, mammalian models are expensive to maintain and have limited scalability. Several non-mammalian models of polyQ toxicity have been developed in organisms such as S.cerevisiae [34], c.elegans [35, 36], and Drosophila [37]. These models are scalable for screening compounds and genetic interactions, but lack high genetic similarity to humans and have significantly different, or in the case of S. cerevisiae no, nervous system. Zebrafish are an advantageous vertebrate model organism that is genetically more closely related to humans than non-vertebrate models but is still scalable and reasonably affordable as compared to mammalian models [38]. HTT-polyQ toxicity has been reported in zebrafish by using mRNA or plasmid DNA injection to acutely over-express the protein $[39,40]$. However, this model might not recapitulate specific mechanisms of the disease due to its early developmental effects and the extreme levels of protein expression that are necessary to cause toxicity. A second zebrafish model of polyQ toxicity has been reported in which the rhodopsin promoter drives mHTT-exon1 fragment expression in photoreceptors of the retina [41]. These zebrafish exhibit specific cellular degeneration and protein aggregation in the rod photoreceptor layer of the retina. However, retinal degeneration is not a known pathology in HD. Therefore, a zebrafish model that more closely recapitulates aspects of the human disease would be a valuable new tool for the field.

We have generated a series of conditional transgenic zebrafish models of HD. Using Cre-loxP technology, we have generated inducible transgenic fish that express HTT-exon1(25Q)-EGFP or mHTT-exon1(97Q)-EGFP 
upon Cre recombination. We have also generated complementary HTT- $\Delta$ N17-exon1(25Q)-EGFP and mHTT$\Delta \mathrm{N} 17$-exon1(97Q)-EGFP lines. These latter models were created to test if the accelerated nuclear pathogenesis and disease-like phenotypes observed originally in BACHD$\Delta \mathrm{N} 17$ mice [28] could also be seen in our zebrafish model, and to test if N17 plays a crucial role in modifying the toxicities of mHTT-exon1, a disease-relevant toxic fragment in HD [32]. Upon ubiquitous recombination, EGFP ${ }^{+}$ protein aggregates are visible within both mHTT-exon1 and mHTT- $\Delta$ N17-exon1 lines. Surprisingly, these fish develop normally up to five weeks of age at which point mHTT- $\Delta$ N17-exon1 lines begin to exhibit abnormal movement and swimming behavior that progressively worsen until the fish are unable to swim by about 12 weeks of age. The mHTT-exon1 lines present much milder swimming impairment that does not appear until 4 months of age and progresses much more slowly. Additionally, we crossed the mHTT- $\Delta$ N17-exon1 line into transgenic Cre driver lines for neurons, glia, muscle, or vasculature. Only fish expressing $\mathrm{mHTT}-\Delta \mathrm{N} 17$-exon 1 specifically in neurons developed a progressive movement disorder. Finally, we examined the subcellular localization of mHTT-exon1 fragments in the transgenic fish and found that mHTT- $\Delta$ N17-exon1 is enriched in the nucleus of neurons, providing direct evidence that N17 is crucial for cytoplasmic targeting/nuclear export of mHTTexon1 in vivo and further suggests the nuclear toxicity is key to the manifestation of disease-like phenotypes in an HD vertebrate model. This new zebrafish model of HD will be an invaluable tool to further dissect the mechanisms of mutant polyQ toxicity and to screen for potential therapeutics or genetic modifiers to ameliorate disease-like phenotypes in vivo.

\section{Results \\ Cre-loxP inducible expression of human HTT exon 1 in transgenic zebrafish}

Since our earlier experiments had demonstrated significant toxicity leading to premature death of transgenic zebrafish upon over-expression of polyQ expanded HTT, we decided to create Cre-loxP conditionally inducible transgenic fish. This system allows us to maintain the transgenic lines by bypassing any toxicity by keeping the transgene switched off until expression of Cre recombinase is introduced, which also allows us spatial and temporal control of expression. We chose to use the strong, ubiquitous bactin2 promoter provided with the tol2kit to drive transgene expression [42]. Each transgene was assembled with the bactin 2 promoter, floxed $m$ Cherry, followed by one of four HTT-exon1 cassettes $(25 \mathrm{Q}, 97 \mathrm{Q}(\mathrm{m}), \Delta \mathrm{N} 17-25 \mathrm{Q}$, or $\Delta \mathrm{N} 17-$ $97 \mathrm{Q}(\mathrm{m})$ ) fused to GFP flanked by tol2 transposon sites (Fig. 1a). The polyQ coding DNA sequences were mutated at wobble sites as reported previously [29] to stabilize the transgene and prevent the genomic instability associated with CAG repeats. Transgenic germlines were established for each transgene, one line was maintained for HTTexon1 and HTT- $\Delta$ N17-exon1 control fish while two separate lines were maintained for mHTT-exon1 and mHTT- $\Delta$ N17-exon1. Transgenic lines have been maintained to at least $\mathrm{F}_{6}$ generation with Mendelian inheritance patterns suggesting a single insertion site is present in each line. Additionally, the use of the Tol2 system should result in single copy transgene insertions. To confirm that these transgenes were inducible by $\mathrm{Cre}$, we injected embryos with Cre mRNA and observed loss of mCherry expression and induction of GFP expression in all lines (Fig. $1 \mathrm{~b}$ and data not shown). HTT-exon1 L1 and HTT- $\Delta$ N17-exon1 L1 lines exhibited ubiquitous, diffuse GFP expression at all time points examined while mHTT-exon1 and mHTT- $\Delta$ N17-exon1 lines initially had ubiquitous expression but exhibited $\mathrm{GFP}^{+}$protein aggregates by 5 days post fertilization (dpf), most prominently in trunk muscles (Fig. 1c). To measure the expression level of each transgene in the different lines, western blots and qRTPCR were performed. Each line was crossed to heat shock inducible Cre (HS:cre) transgenic fish and then heat shocked at shield stage resulting in robust, ubiquitous recombination. To evaluate protein levels, Western blots were performed on pools of embryos from three separate clutches harvested at $5 \mathrm{dpf}$ for the HTT-GFP fusion protein and $\alpha$-tubulin as a loading control (Fig. 1d, upper panel). Densitometry was used to measure relative protein abundance (Fig. 1d, lower panel). Embryos lacking HS:cre never show GFP expression (data not shown). HTT-exon1 L1 and HTT- $\Delta$ N17-exon1 L1 both exhibit strong GFP expression upon recombination at a size slightly larger than the predicted $\sim 37 \mathrm{kDa}$ and $\sim 35 \mathrm{kDa}$, suggesting post-translational modification was present. Similarly, both mHTT-exon1 and mHTT- $\Delta$ N17-exon1 appear at sizes larger than their predicted $\sim 46 \mathrm{kDa}$ and $\sim 44 \mathrm{kDa}$ molecular masses, which may result from aberrant conformation due to the expanded polyQ and/or post-translational modification (Fig. 1d, upper panel). mHTT-exon1 L1 and L2 exhibit similar levels of expression that are significantly lower than line HTT-exon1 L1, HTT- $\Delta$ N17-exon1 L1, and both mHTT- $\Delta$ N17-exon1 lines ( $p<0.01$, ANOVA, Bonferroni posthoc test) (Fig. 1d). mHTT- $\Delta$ N17-exon1 L1 and L2 also exhibit similar levels of expression that are lower than HTT- $\Delta$ N17-exon1 L1 but with only L2 reaching statistical significance $(p<0.01$, ANOVA, Bonferroni posthoc test) (Fig. 1d). We were surprised to observe the lower protein levels for the mHTT-exon1 lines as compared to the mHTT- $\Delta$ N17-exon1 lines since the transgene design and promoter were identical. To test whether transgene mRNA expression was responsible for the difference, we performed qRT-PCR analysis on mRNA extracted from each line. For this analysis, pools of ten $\mathrm{GFP}^{+}$embryos from six 


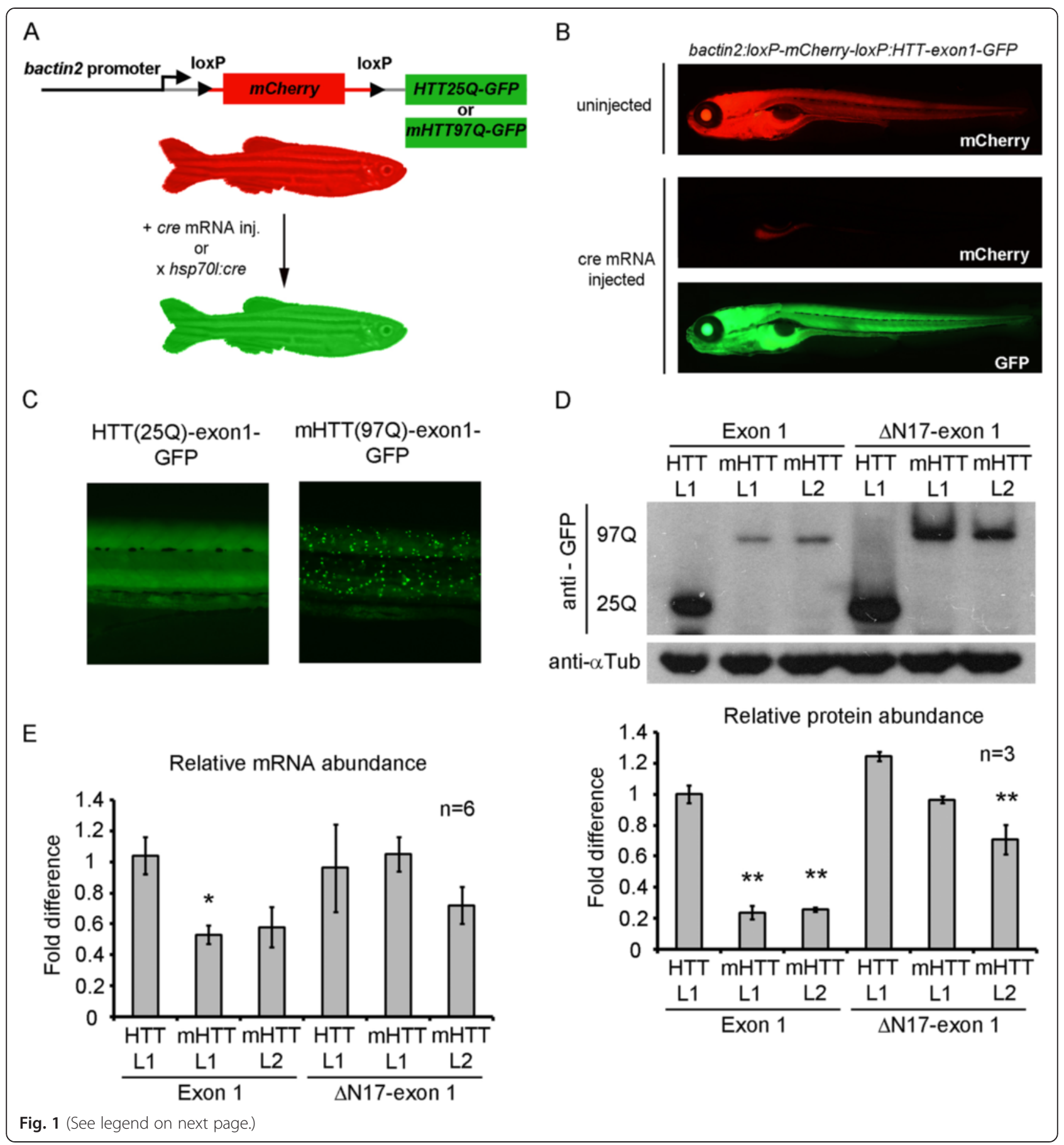


(See figure on previous page.)

Fig. 1 Design and demonstration of Cre-loxP inducible HTT-exon 1-GFP expression in transgenic zebrafish. a Schematic of the transgene design using the bactin2 promoter to drive expression of floxed mCherry followed by the HTT-exon 1 cassette being tested. Following Cre expression by mRNA injection or HS:cre transgene induction, mCherry is recombined out and the desired HTT-exon 1 cassette fused to GFP is expressed ubiquitously. b Photo micrographs depicting 5 day old, HTT-exon1(25Q) embryos displaying mCherry expression when Cre is absent (upper panel) or mCherry negative, HTT-exon1-GFP positive expression following cre mRNA injection (bottom two panels). c Close-up view of the trunk of HTT(25Q)-exon1-GFP and mHTT(97Q)-exon1-GFP embryos demonstrating ubiquitous, diffuse expression of HTT-exon1-GFP (left panel) and ubiquitous, aggregated expression of mHTT-exon1-GFP (right panel). d A representative western blot and densitometric measurments for each transgenic line $(n=3)$. Anti-GFP antibody (top panel) and anti-tubulin loading controls (bottom panel) on protein isolated from pools of 5 day old embryos for each HTT transgenic line crossed to HS:cre. $97 \mathrm{Q}$ and $25 \mathrm{Q}$ mark the location of mHTT-GFP and HTT-GFP protein bands respectively. Protein expression was highest in HTT-exon1 L1 and HTT- $\Delta \mathrm{N} 17$-exon1 L1 lines. mHTT-exon1 line $1(\mathrm{~L} 1)$ and line 2(L2) both express at similar levels as do mHTT- $\Delta \mathrm{N} 17$-exon1 line 1(L1) and line 2(L2). Densitometric measurements (lower bar graph) demonstrate significantly lower levels of protein in $\mathrm{mHTT}$-exon1 L1 and L2 compared to all other lines while $\mathrm{mHTT}-\Delta \mathrm{N} 17$-exon $1 \mathrm{~L} 2$ is significantly lower than HTT-exon1 L1 and HTT- $\Delta$ N17-exon1 L1. Comparisons were done by one-way ANOVA with Bonferroni posthoc test, ${ }^{* *} p<0.01$, error bars are SEM. e Quantification of transgene expression by quantitative RT-PCR. Six pools of ten embryos from separate clutches of each HTT transgenic line crossed to HS:cre were analyzed. Comparisons of expression level were done by one-way ANOVA with Bonferonni posthoc test, ${ }^{*} p<0.05$, error bars are SEM

independent clutches were assayed for each line. mRNA levels were less varied between lines than protein, with mHTT-exon1 L1 the only one to reach statistical significance in comparison to HTT-exon1 L1 ( $p<0.05$, ANOVA, Bonferroni posthoc test) (Fig. 1e). It is important to note that mRNA levels between mHTT-exon1 lines and mHTT- $\Delta$ N17-exon1 lines are similar. This is in contrast to the protein levels, suggesting that post-translational mechanisms may be affecting protein abundance. In fact, the N17 region of HTT has been described as a modifier of protein stability, harboring both phosphorylation [26, 27] and ubiquitination [17] sites that regulate protein degradation. Therefore, it appears that the intact N17 domain leads to greater protein instability in this zebrafish model and its deletion accelerates protein accumulation.

\section{The transgenic zebrafish expressing $\mathrm{mHTT}$-exon1 lacking the $\mathrm{N} 17$ domain exhibits accelerated, progressive movement disorder}

Each HTT transgenic line was crossed with HS:cre and the embryos heat shocked at shield stage to induce recombination or left as non-heat shocked controls. HTTexon1 L1 $(n=21)$ and HTT- $\Delta$ N17-exon1 L1 $(n=26)$ both exhibited robust GFP expression and developed normally with no behavioral changes up to 26 weeks of age (Fig. 2a and c and Additional file 1: Movie S1 and Additional file 2: Movie S2). mHTT-exon1 L1 $(n=16)$ and L2 $(n=16)$ both survived early development even though mHTT-exon1 protein aggregates were present by $5 \mathrm{dpf}$. These fish behaved normally until 16 weeks of age, at which point mild behavioral abnormalities, such as abnormal swimming and immobility alternating with jerky movement, were noted in a small number of fish in mHTT-exon1 L1 with more fish displaying these behaviors over time (Fig. 2c). mHTT L2 has not displayed any behavioral abnormalities up to 32 weeks of age. mHTT- $\Delta$ N17-exon1 L1 $(n=10)$ and L2 $(n=24)$, in contrast, developed a robust, progressive movement deficit (Fig. 2b and c, and Additional file 3: Movie S3 and Additional file 4: Movie S4). mHTT-exon1, mHTT- $\Delta$ N17-exon1 L1, and mHTT- $\Delta$ N17-exon1 L2 all exhibit statistically significant differences in survival curve analysis as compared to each other as well as HTT-exon1 L1, HTT- $\Delta$ N17-exon1 L1, and mHTT-exon1 L2 (Fig. 2c, Kaplan Meier with Log Rank, $p<0.001)$. We grouped the observed behaviors into three stages according to their severity (Fig. 2d): Stage 1 - abnormal swimming, immobility alternating with jerky movement; Stage 2 - loss of lateral stability, corkscrew swimming (see Fig. 2b); Stage 3 - loss of vertical stability, inability to coordinate swimming, death. mHTT-exon1 L1 fish begin exhibiting symptoms at 16 weeks and slowly progress so that by 44 weeks $50 \%$ of the fish have reached Stage 3 (Fig. 2e). However, $40 \%$ of the fish are still unaffected. mHTT- $\Delta$ N17-exon1 L1 begins exhibiting Stage 1 symptoms by 5 weeks of age and progresses to Stage 3 by 10-12 weeks (Fig. 2f). mHTT$\Delta$ N17-exon1 97Q L2 does not exhibit Stage 1 symptoms until 8 weeks and progresses more slowly with Stage 3 not reached for all fish until after 20 weeks (Additional file 5). These experiments have been repeated in multiple generations with similar results. Non-heat shocked control transgenic fish for all lines, including both mHTT- $\Delta$ N17-exon1 lines, develop normally and do not show abnormal movement before 1 year of age (the last observation made), although some leaking recombination was noted. Overall, we have found that mHTT- $\Delta$ N17-exon1 transgenic fish consistently exhibit a progressive movement deficit, while mHTTexon1 fish show a later-onset and more slowly progressing behavioral impairment. Importantly, the control HTT-exon1 and HTT- $\Delta$ N17-exon1 fish are indistinguishable from wild-type fish. Since the phenotypes of the transgenic fish were analyzed up to 11 months of age, it is possible that the mHTT-exon1 zebrafish could develop more severe disease-like phenotypes as they age. 


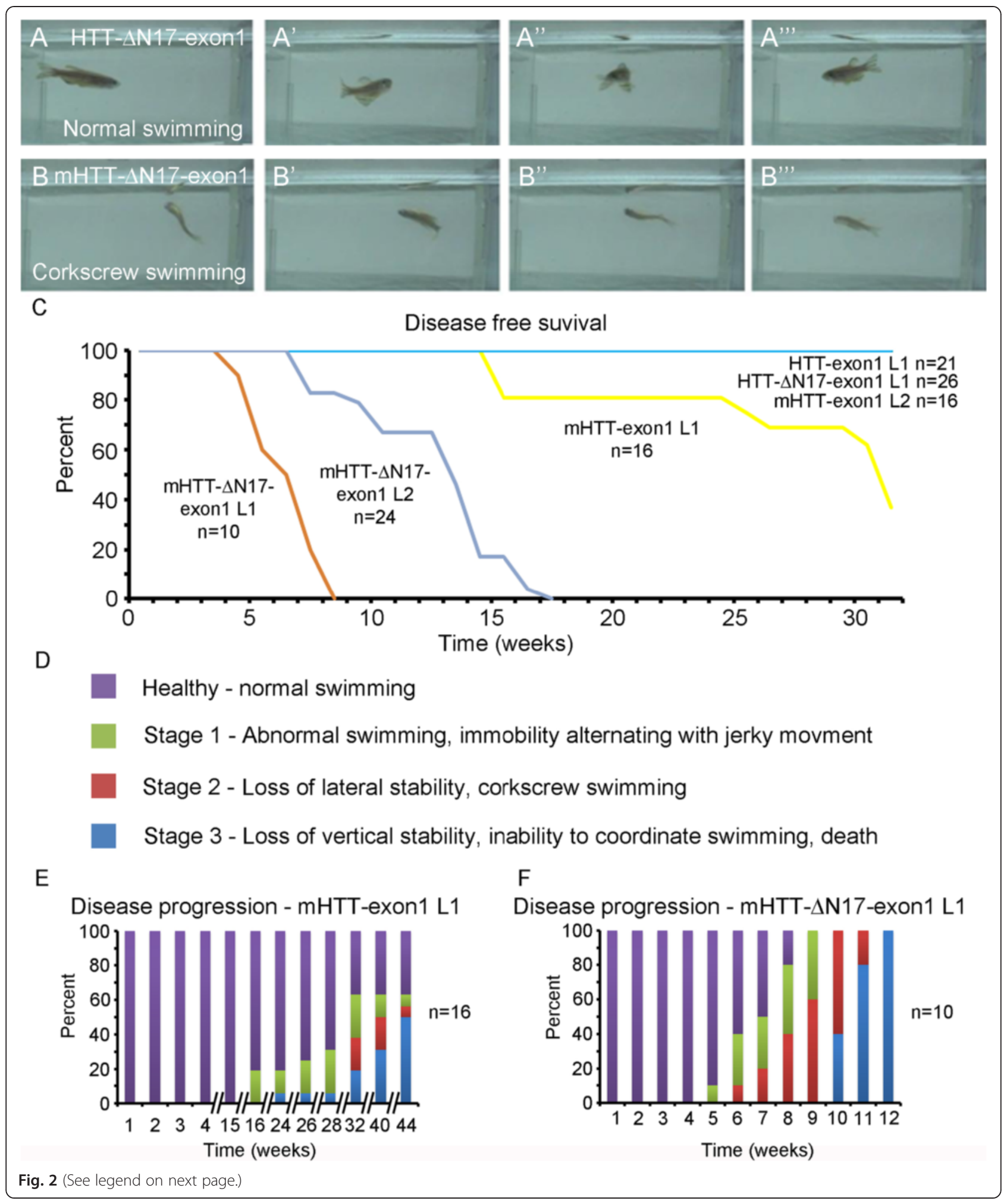




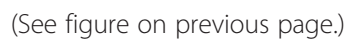

Fig. 2 mHTT- $\triangle N$ 17-exon1 transgenic fish develop a progressive motor behavior phenotype. a Panels taken from Additional file 1: Movie S1 showing normal swimming behavior in a HTT- $\Delta$ N17-exon1 transgenic fish. b Panels taken from Additional file 3: Movie S3 showing abnormal swimming behavior, corkscrew swimming, in a $\mathrm{mHTT}-\Delta \mathrm{N} 17$-exon1 transgenic fish. c Disease free survival curve for each HTT-exon1 transgenic line corresponding to disease onset. Note that $\mathrm{mHTT}-\Delta \mathrm{N} 17-\mathrm{exon} 1 \mathrm{~L} 1 \quad(n=10)$ and L2 $(n=24)$ both develop symptoms earlier than mHTT-exon1 L1 $(n=16)$ and that mHTT-exon1 L2 $(n=16)$, HTT-exon1 $(n=21)$, and HTT- $\Delta$ N17-exon1 $(n=26)$ do not exhibit symptoms in the observed time frame. Kaplan Meier analysis with Log Rank test, $p<0.001$ for $\mathrm{mHTT}-\Delta \mathrm{N} 17$-exon1 L1, mHTT- $\Delta \mathrm{N} 17$-exon1 L2, and mHTT-exon1 L1. d Behavioral characterization within different transgenic lines. Behavior was grouped into four categories: Healthy, Stage 1, Stage 2, or Stage 3 as described. Observations were made weekly. e Disease progression of mHTT-exon1 L1 fish. Note the discontinuous X-axis to account for the extended time frame of behavioral changes $(n=18)$. $\mathbf{f}$ Disease progression of $\mathrm{mHTT}-\Delta \mathrm{N} 17-\mathrm{exon} 1 \mathrm{~L} 1$. $\mathrm{mHTT}-\Delta \mathrm{N} 17-\mathrm{exon} 1 \mathrm{~L} 1$ fish developed a robust, progressive motor behavioral deterioration beginning at $5-8$ weeks of age and progressing to immobility and death by 12 weeks $(n=10)$

\section{The transgenic zebrafish expressing $\mathrm{mHTT}-\Delta \mathrm{N} 17-$-exon1 exhibit reduced brain weight}

To examine if brain atrophy, a hallmark of neuropathology in HD, was present in the mHTT- $\Delta \mathrm{N} 17$-exon1 fish, brain weight from Stage 3 fish was compared to nonheat shocked fish and control HTT- $\Delta$ N17-exon1 with and without heat shock. Fish from each group were age matched and raised under equal population density and tank size. This is an important consideration for zebrafish since they exhibit density dependent growth [43]. The average body length and weight of each group of fish was not significantly different from each other $(n=8$ for each group) (Fig. 3, a and b). The zebrafish brains from each line (excluding the olfactory bulbs and eyes) were dissected and weighed. Interestingly, heat shocked, Stage 3 mHTT- $\Delta$ N17-exon 1 fish exhibited a significantly smaller brain weight than the brains from non-heat shocked mHTT- $\Delta$ N17-exon1 fish or HTT- $\Delta$ N17-exon1 fish with or without heat shock (Fig. 3c) (ANOVA with Bonferroni post hoc, $p<0.05)$. This result suggests that mHTT- $\Delta$ N17-exon1 fish exhibit brain atrophy similar to that seen in HD patients.

\section{N17 domain determines the cytoplasmic versus nuclear accumulation of mutant HTT exon 1 fragments in vivo}

The 17N-terminal amino acids of HTT have been reported to regulate protein stability, toxicity, and subcellular localization. In a mouse BACHD model with N17 deleted in the context of the full length HTT gene, dramatically accelerated and exclusive nuclear accumulation of mHTT fragments were demonstrated [28]. In the context of mHTT-exon1, an HD-relevant pathogenic fragment [32], N17 is necessary for nuclear export/cytoplasmic targeting in vitro $[44,45]$ : its relevance in mediating nuclear versus cytoplasmic pathology in vivo remains unclear. Therefore, we assessed if the localization of aggregates was different in transgenic HD fish expressing mHTT-exon1 with or without intact N17. First, we immunostained brain sections of Stage 3, HS:crel mHTT- $\Delta$ N17-exon1 fish, HS:cre/HTT- $\Delta$ N17-exon1 fish, HS:cre/mHTT-exon1 fish, and HS:cre/HTT-exon1 fish with the anti-human HTT antibody S830 [46] (Fig. 4a-d). HTT- $\Delta$ N17-exon1 and HTT fish exhibit diffuse staining throughout the brain with no visible aggregates (Fig. $4 \mathrm{a}$ and $\mathrm{c}$ ). mHTT- $\Delta \mathrm{N} 17$-exon 1 and mHTT-exon1 fish both exhibit robust aggregate staining throughout the brain (Fig. 4b and d). The aggregates in mHTT-exon1 appear as small puncta with occasional larger accumulations (Fig. 4b) while the aggregates in mHTT- $\Delta$ N17-exon1 appear mainly as large oval shaped staining reminiscent of nuclei (Fig. 4d).

To examine the cellular and subcellular localization of these aggregates in more detail, we combined immunofluorescent staining for neurons (anti-HuC) with GFP staining of the HTT-exon1 fusion protein and DAPI for nuclei. We focused our examination on the ventrolateral nucleus of torus semicircularis, a brain region with strong $\mathrm{HuC}$ staining and a mixture of cell bodies and neuropil. In HTT-exon1 and HTT- $\Delta \mathrm{N} 17-$-exon1 fish, diffuse GFP staining was present throughout the brain including neurons (Additional file 6). Close examination by confocal microscopy demonstrated that HTT-exon 1 is largely excluded from the nucleus while HTT- $\Delta$ N17exon1 is detectible in the nucleus suggesting the nuclear export function of N17 is intact in vivo (Additional file 6). mHTT-exon1 fish exhibited robust aggregate formation, with the majority localized away from $\mathrm{HuC}$ positive neuronal cell bodies (Fig. 4e-h). These aggregates are likely present in the neuropil (e.g. axons and dendrites), but it is also possible that a subset of them are present in nonneuronal cells. Occasional $\mathrm{GFP}^{+}$aggregates are present in the peri-nuclear regions stained by $\mathrm{HuC}$ or weakly and diffusely in DAPI positive nuclei (Fig. $4 \mathrm{e}-\mathrm{h}$, arrowheads highlight $\mathrm{GFP}^{+}$nuclei). mHTT- $\triangle \mathrm{N} 17$-exon1 fish, on the other hand, exhibited strong and predominant nuclear accumulation of mHTT- $\Delta$ N17-exon1 that was found almost exclusively in the $\mathrm{HuC}$ positive neurons (Fig. 4i-1). Neuropil aggregates similar to those seen in mHTT-exon1 fish were also present, but were much less abundant. 3D confocal projections clearly demonstrated the neuropil localization of HTT aggregates in mHTT-exon1 fish (Fig. $4 \mathrm{~m}$ ) and nuclear accumulation in the mHTT- $\Delta \mathrm{N} 17$ exon1 fish (Fig. 4n). Quantification of HTT-GFP location demonstrates significant enrichment of nuclear mHTT$\Delta \mathrm{N} 17$-exon1 in $\mathrm{HuC}$ positive neurons of mHTT- $\Delta \mathrm{N} 17-$ exon1 fish (Fig. 4o, $p<0.05$, ANOVA with Bonferonni 


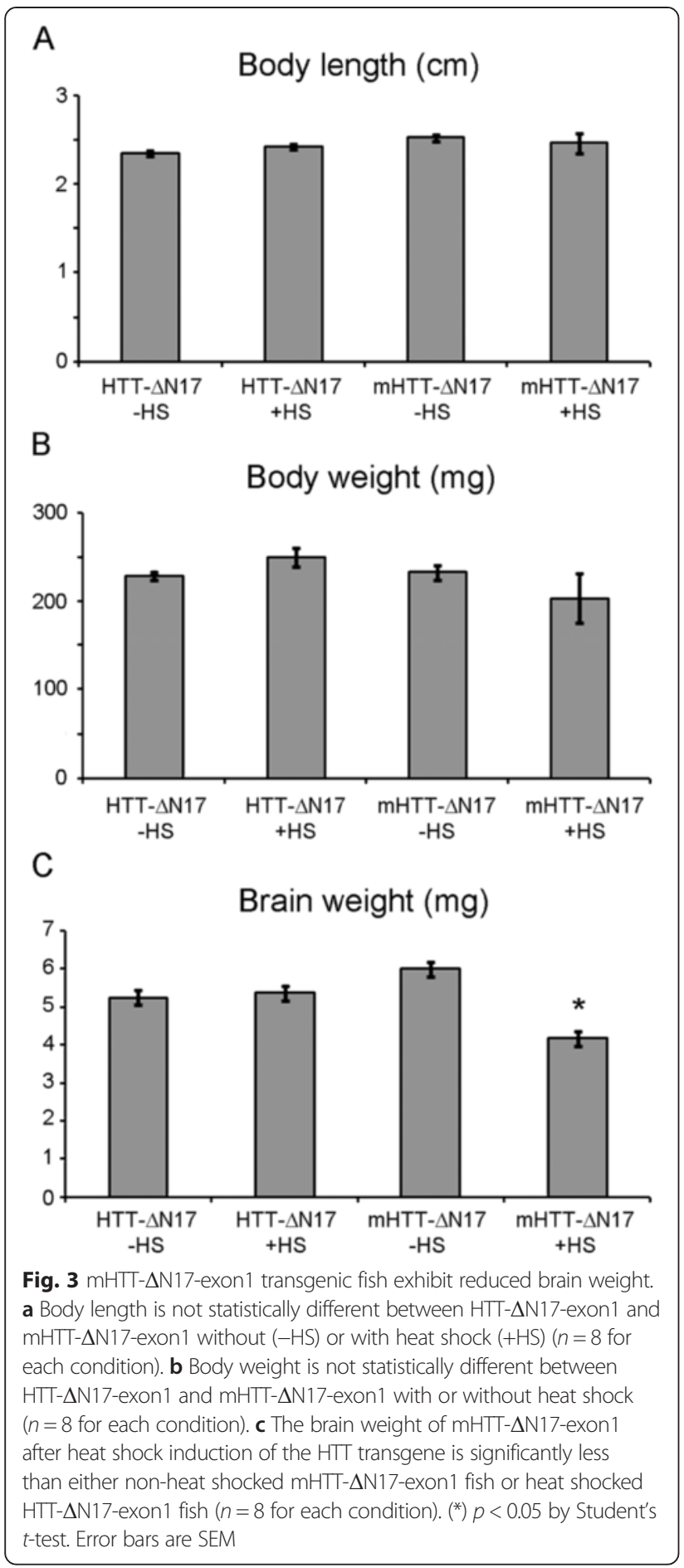

post hoc). No difference was detected in cell density, percent $\mathrm{HuC}$ positive cells, or aggregate density (nuclear accumulation was scored as a single aggregate) in mHTTexon1 versus mHTT- $\Delta$ N17-exon1 fish (Additional file 7). Taken together, our study is consistent with the in vitro studies in cells and mouse study with BACHD- $\triangle \mathrm{N} 17$ mice that N17 is a crucial element in preventing nuclear pathogenesis for mHTT-exon1, and our study provides the first in vivo evidence that N17 can prevent nuclear accumulation and aggregation of a known pathogenic mHTT fragment, mHTT-exon1, in a vertebrate model of HD.

\section{Mutant HTT exon1 lacking the N17 domain exerts toxicity selectively in neurons to induce movement deficits in HD transgenic zebrafish}

HD is generally thought to be caused by neuron specific dysfunction and atrophy. However, since HTT expression is ubiquitous it is possible that disease specific pathologies are occurring in other cell types. HTT-polyQ expression in glia [47], immune cells [48], skeletal muscle [49] and vasculature $[50,51]$ have all been suggested to contribute to $\mathrm{HD}$. A recent study using conditional BAC transgenic mouse models of HD (BACHD) revealed distinct and synergistic roles of full-length mHTT expressed in cortical pyramidal neurons and striatal MSNs in eliciting multiple behavioral deficits and selective neurodegeneration [52]. However, these prior models lack the overt and progressive movement disorder seen in our mHTT- $\Delta$ N17-exon1 fish model, and it is unclear if mHTT-exon1 lacking N17 is ubiquitously toxic or may retain pathogenic specificity similar to the intact mHTT-exon1. To begin answering such a question, we crossed $\mathrm{mHTT}-\Delta \mathrm{N} 17$-exon1 fish with Cre driver lines with selective Cre expression in lineages for neurons (elavl3-Cre) [53], glia (gfap-Cre) [54], skeletal muscle (mylpfa-Cre) [55], and endothelial (etv2-Cre) [56] cells. Each cross gave expected tissue and cell-type specific mHTT-exon 1 transgene expression (Fig. 5a-d). GFP ${ }^{+}$fish from each cross were selected and raised for behavioral observation ( $n=>20$ for each genotype). Only the neuron specific, elavl3:cre/mHTT- $\Delta$ N17-exon1 fish developed movement abnormalities reminiscent of the ubiquitously activated mHTT- $\Delta$ N17-exon1 (Fig. 5e, $p<0.001$, Kaplan Meier analysis). Using our previously described behavioral categories, we found that elavl3:cre/mHTT- $\Delta \mathrm{N} 17$-exon1 fish exhibit all of the progressively deteriorating movement behaviors described in the ubiquitously expressing fish (Fig. 5f). It should be noted that the movement abnormalities observed in elavl3:cre/mHTT- $\Delta$ N17-exon1 fish appeared and progressed more slowly than ubiquitously expressing fish, suggesting the possibility that nonneuronal cells modify the effect of mHTT on neurons in this model.

To examine the behavior of these fish in a more quantitative manner, we performed field potential recordings [57] of freely behaving elavl3:cre/mHTT- $\Delta$ N17-exon1 $(n=6)$ and elavl3:cre/HTT $\Delta \mathrm{N} 17$ fish $(n=6)$ fish (Fig. 6). elavl3:cre/mHTT- $\Delta$ N17-exon1 fish were categorized as Stage 1 at the time of this experiment while elavl3:cre/HTT- $\Delta$ N17-exon1 fish were categorized as healthy. Field potential recordings are performed by placing individual fish into a small chamber with recording 

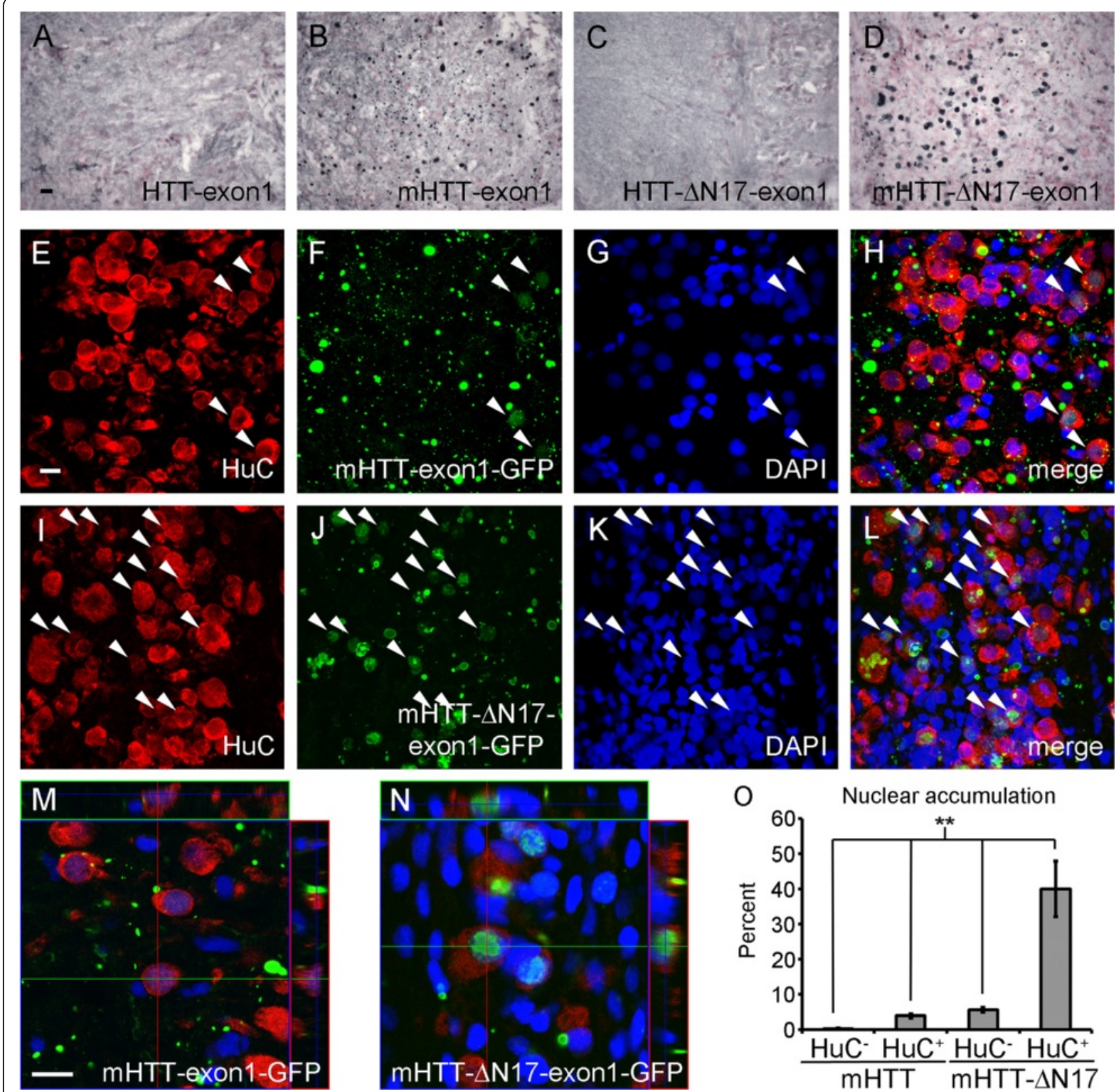

Fig. $4 \mathrm{mHTT}-\Delta \mathrm{N} 17$-exon1 accumulates mainly in the nucleus of neurons while $\mathrm{mHTT}$-exon1 aggregates are mostly outside the neuronal cell body. Brain sections from 26 week old HTT-exon 1 and $m H T T$-exon 1 fish or 12 week old HTT- $\Delta \mathrm{N} 17$-exon 1 and $m H T T-\Delta \mathrm{N} 17$-exon1, Stage 3 fish were immunostained to observe the transgenic HTT-exon1 localization. All images are from the hindbrain region and similar staining was present throughout all brain regions in each fish. a-d 5830 anti-human HTT Exon 1 antibody was used to detect transgene expression in each transgenic line. HTT-exon1 (a) and HTT- $\Delta N 17$-exon1 (c) tissue exhibits uniform, ubiquitous expression. mHTT-exon1 (b) tissue displays many small but distinct aggregates while $\mathrm{mHTT}-\Delta \mathrm{N} 17$-exon1 (d) tissue has many large aggregates. Scale bar equals $25 \mu \mathrm{m}$. e-I Immunofluorescent staining of mHTT-exon1 $(\mathbf{e}-\mathbf{h})$ and $\mathrm{mHTT}-\Delta \mathrm{N} 17-\mathrm{exon} 1(\mathbf{i}-\mathbf{I})$ transgenic fish for neurons (HuC), transgenic HTT-exon1-GFP fusion protein, and nuclei (DAPI). mHTT-exon1 tissue exhibits many small GFP ${ }^{+}$aggregates that are not generally associated with HuC positive neuronal cell bodies suggesting they are either in the axons or dendrites or are non-neuronal (e-h). Occasional, weak nuclear accumulation is present in a few neurons (white arrowheads). mHTT- $\Delta \mathrm{N} 17-\mathrm{exon} 1$ tissue has many neuronal, HuC positive, nuclei co-localized with HTT-exon1-GFP (i-I). Scale bar equals $10 \mu \mathrm{m}$. $\mathbf{m}$ and $\mathbf{n}$ 3D confocal projections of mHTT-exon1 (m) and mHTT- $\Delta$ N17-exon1 (n) tissue demonstrating weak nuclear HTT-exon1-GFP staining in mHTT-exon1 and strong nuclear staining in $\mathrm{mHTT}-\Delta \mathrm{N} 17$-exon1. Scale bar equals $10 \mu \mathrm{m}$. o Quantification of protein accumulation location. Nuclear HTT-exon1 aggregates were rare in HuC negative cells of both lines and $\mathrm{mHTT}$-exon1 transgenic HuC positive neurons. $\mathrm{mHTT}-\Delta \mathrm{N} 17$-exon1 transgenic fish exhibited robust nuclear accumulation of HTT-exon1-GFP in $\sim 40 \%$ of HuC positive cells. ANOVA with Bonferroni posthoc test, $\left.{ }^{(*}\right) p<0.01$ 

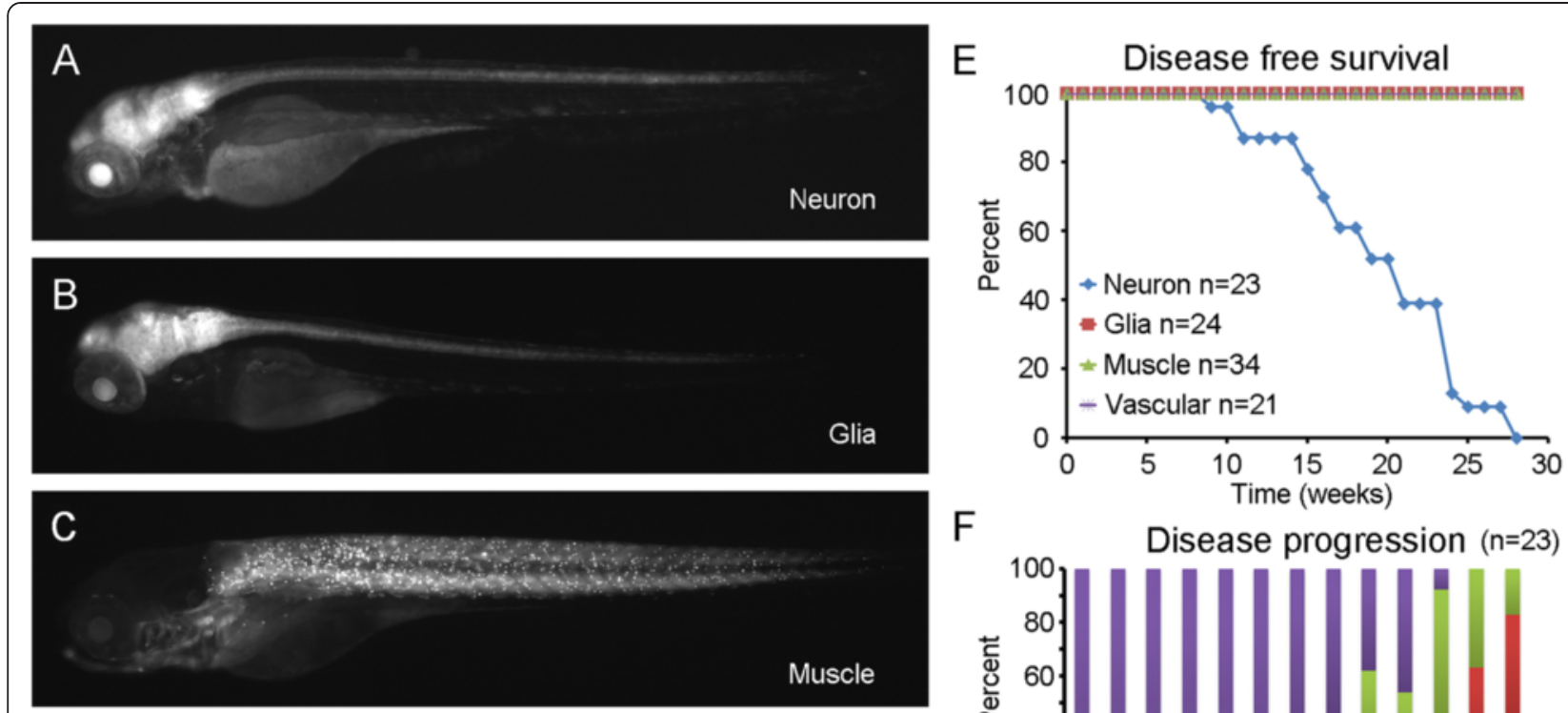

$\mathrm{F}$
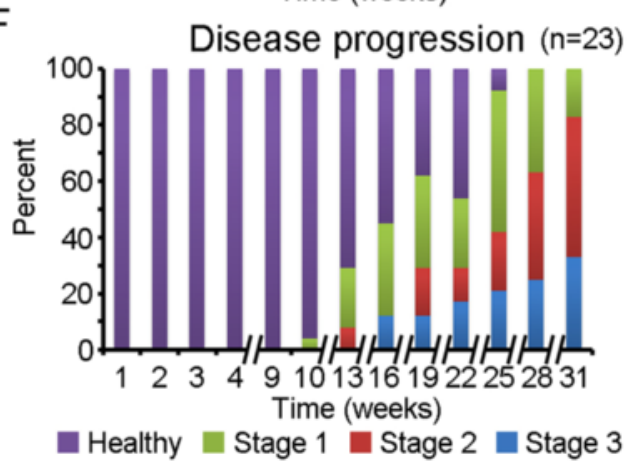

Fig. 5 Neuron specific activation of $\mathrm{mHTT}-\Delta \mathrm{N} 17$-exon1 causes motor behavioral phenotype. a elav/3:cre $(n=23)$, b gfap:cre $(n=24)$, c $m y / p f a: c r e$ $(n=34)$, and $\mathbf{d}$ etv2:cre $(n=21)$ transgenic lines were incrossed to $\mathrm{mHTT}-\Delta \mathrm{N} 17$-exon1 to activate the transgene specifically in neurons, glia, muscle, and vascular cell lineages respectively. GFP fluorescence in living embryos demonstrates the spatially restricted expression of each line at 5 days post fertilization. e A disease free survival curve demonstrating that only elav/3:cre/mHTT- $\Delta \mathrm{N} 17$-exon1 fish develop a behavioral phenotype similar to ubiquitous activation of $\mathrm{mHTT}-\Delta \mathrm{N} 17$-exon1, $p<0.01$, Kaplan Meier survival analysis. $\mathbf{f}$ Disease progression of elav/3:cre/ $\mathrm{mHTT}-\Delta \mathrm{N} 17-\mathrm{exon} 1 \mathrm{fish}$ is similar to ubiquitious $\mathrm{mHT}-\Delta \mathrm{N} 17-\mathrm{exon} 1$ fish although temporally delayed, note the discontinuous $\mathrm{x}$-axis $(n=23)$. Behavioral Stages are described in Fig. 2

electrodes at each end. When a fish moves the electrical activity of the muscle creates a potential across the electrodes that can be recorded over time. This gives an indirect measurement of each fish's motor activity. elavl3:cre/HTT- $\Delta$ N17-exon1 fish exhibit short bursts of activity corresponding to swimming and turning movements within the chamber (Fig. 6a, top panel). elavl3:cre/ mHTT- $\Delta$ N17-exon1 fish exhibited bouts of prolonged activity (Fig. 6a bottom panel and c). However, elavl3:crel mHTT- $\Delta$ N17-exon1 fish initiated movements less frequently than elavl3:cre/HTT- $\Delta$ N17-exon1 fish (Fig. 6b). Overall, elavl3:cre/HTT- $\Delta$ N17-exon1 and elavl3:cre/ mHTT- $\Delta$ N17-exon1 fish did not differ in the total amount of time they were active during these trials (Fig. 6d). These results support the behavioral observations that Stage 1 fish exhibit bouts of jerky movement followed by inactivity.

In summary, our cell-type-specific expression of mHTT- $\Delta$ N17-exon1 revealed a remarkable specificity of toxic mHTT fragments to neurons while its toxicity to several non-neuronal cell types including glia, muscle and blood vessels are unremarkable. This result suggests the neurons are particularly sensitive to the nuclear polyQ toxicity in the context of mHTT-exon1, and the presence of N17 domain can delay such toxicity in vivo.

\section{Discussion}

The molecular mechanisms causing HD have been difficult to identify even though the specific gene mutation has been known for 20 years. Many research models have been created in diverse organisms such as rats [58], mice $[29,33,59]$ zebrafish $[39,40]$, fly [37], worm [35, 36], and yeast [34]. Mammalian models have been extremely valuable in validating disease mechanisms and testing candidate therapeutics [30]; they also have limitations such as lack of overt disease phenotypes and limited scalability due to the cost and effort to maintain a large rodent colony. Invertebrate models are highly scalable but lack close genetic homology to humans. The zebrafish model is an ideal compromise between the scalability of invertebrate models and genetic homology to vertebrates. Previously developed zebrafish models of HD are not ideal models of the disease due to lethality caused by high level overexpression of toxic mHTT 


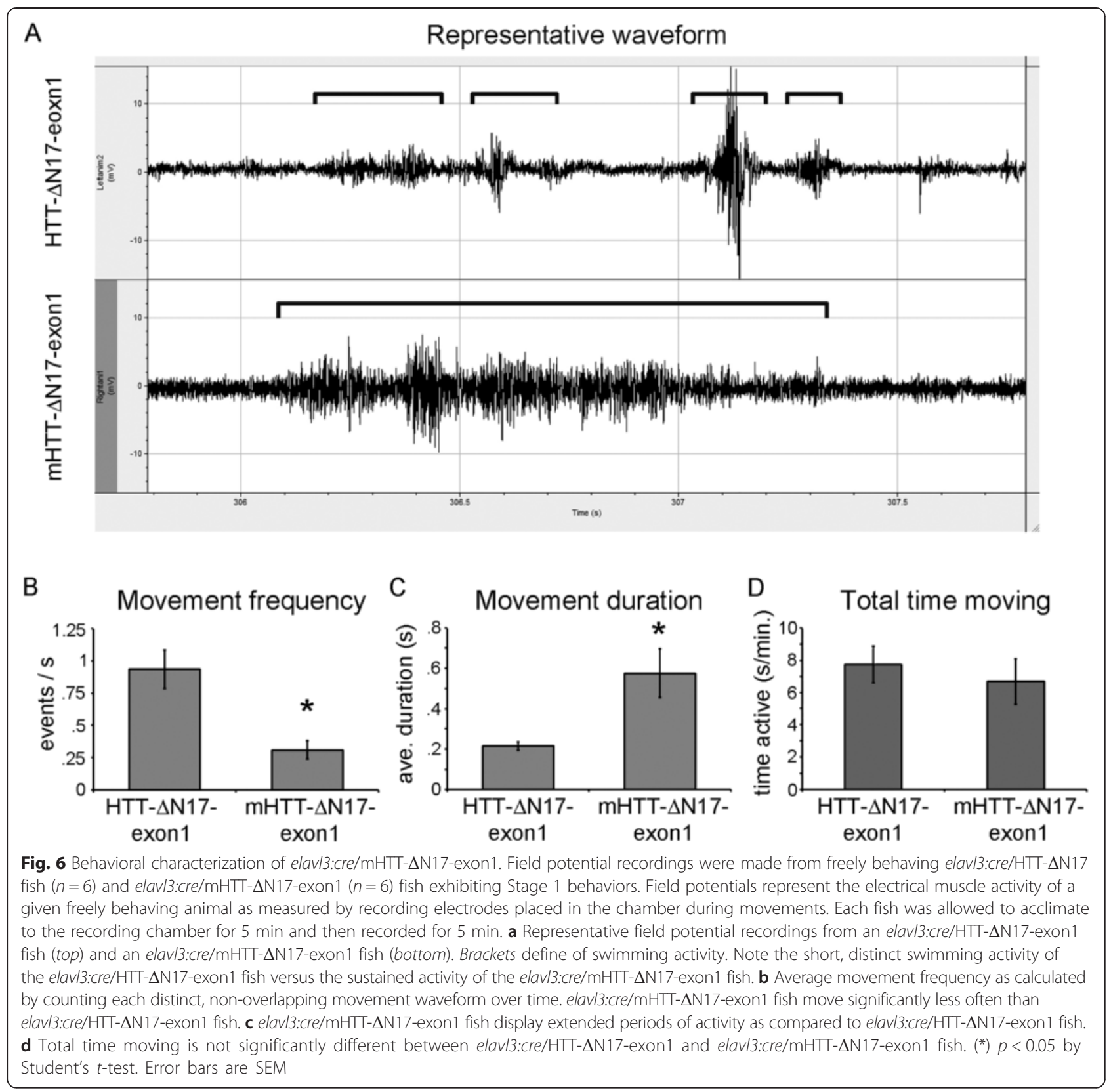

fragments in embryos or limited disease relevance due to restricted tissue expression.

Here we present a novel Cre-loxP inducible zebrafish model of HD. This model allows for precise spatial and temporal control of expression of the disease linked protein, which was previously only available in the mouse $[52,60]$. These transgenic lines can be maintained in non-recombined form and experimental clutches of embryos generated as needed by Cre mRNA injection or intercross with Cre driver transgenic lines. By incrossing tissue specific Cre driver lines to the mHTT- $\Delta$ N17exon1 line we found that neurons appear to be the main cell involved in the abnormal movement behaviors originally observed with ubiquitous expression of this particular toxic polyQ protein. This is in agreement with findings in conditional mouse HD models $[52,60,61]$. Future work will be necessary to establish the specific neuronal population responsible for the behavioral phenotype in this zebrafish model using neuronal sub-type-specific Cre driver lines. Additionally, careful cell-type specific analysis for histological and molecular markers of HD-like pathologies will be necessary to further define the similarities to human disease.

These transgenic fish are based upon expression of mHTT-exon1. In recent years it has become apparent that the exon 1 fragment of the much larger HTT 
protein not only contains the expanded polyQ region that is mutated in the disease but is also a known pathogenic species in the diseased as a result of aberrant mHTT splicing event [32] and proteolysis [31]. A surprising finding in our study is that zebrafish is somewhat refractory to the toxicity of ubiquitously expressed mHTT-exon 1 , since only a few transgenic mHTT-exon 1 fish developed abnormal movements by 6 months of age. Histological examination of these fish demonstrated robust mHTT-exon1-GFP positive protein aggregates formed in the brain, and most of these aggregates are located in the neuropil (e.g. axons, dendrites). One leading hypothesis is that the nucleus is the site of pathological action of expanded polyQ in all polyQ disorders [4]. It has been reported that the N17 terminal amino acids of HTT contains a nuclear export signal $[45,62]$. Additionally, these amino acids have been reported to function in modifying aggregate formation and protein stability [17, 23, 24, 26, 27]. Recently, Gu et. al. [28] showed that deletion of these 17 amino acids in the context of BAC transgenic mice harboring the full length human HTT gene with 97Q causes an adult-onset progressive movement disorder and robust striatal neurodegeneration. Interestingly, these BACHD- $\Delta \mathrm{N} 17$ mice also presents with dramatic acceleration of selective nuclear mHTT aggregates due to accumulation of small mHTT polyQ fragments. We therefore, tested whether deletion of these 17 amino acids in the context of an HTT exon 1 based transgene would cause accelerated disease in our zebrafish model. Mutant HTT- $\Delta$ N17-exon1 expression caused a rapidly developing progressive movement disorder that ultimately led to early death. The development of this behavioral phenotype was correlated with massive accumulation of mHTT- $\Delta$ N17-exon1-GFP in the nucleus of neurons. Interestingly, although expression was ubiquitous, large nuclear aggregates were only observed in neurons, suggesting mechanisms of nuclear accumulation and aggregation of the mutant polyQ protein is neuronal selective. We did not observe robust nuclear accumulation of the non-toxic HTT- $\Delta$ N17-exon1 transgene suggesting that lack of $\mathrm{N} 17$ function alone without polyQ expansion is not sufficient to elicit high levels of nuclear protein accumulation. Our study confirmed and extended the results found in BACHD- $\triangle \mathrm{N} 17$ mice by demonstrating not only that N17 is crucial in preventing nuclear mHTT toxicity and onset of disease-like phenotypes in an vertebrate, but also provide strong evidence that N17 is crucial in mitigating the ability of mHTT-exon1 fragment, a known pathogenic species in HD, to mediate its nuclear toxicity and disease pathogenesis in an intact vertebrate model.

A truly surprising finding in our study is that the mHTT- $\Delta$ N17-exon1 fragment elicits robust and progressive movement disorder through its toxicity in neurons, but not in astrocytes, skeletal muscles, or vascular cells. This result showed that neurons, unlike the non-neuornal cells, may have selective impairment in managing the proteostasis of the expanded polyQ proteins, especially when such protein is translocated and accumulated in the nucleus. Our result is consistent with the rich evidence that expanded polyQ protein is neurotoxic in an artificial context of an endogenous protein [63], or other polyQ diseases (e.g. SBMA or SCA1) in which nuclear translocation is essential to disease pathogenesis [4]. Our study has important implications in the context of understand HD pathogenesis and therapy. First, it suggests that despite the fact that mHTT-exon1 is naturally occurring and is more toxic than longer mHTT-exon1 fragments or full-legnth mHTT, the N17 domain can still provide substantial protection against its ability to translocate into the nucleus and elicit severe disease. Second, since in HD patients and HD mice mHTT fragments (including exon 1 fragment) eventually accumulate in the nucleus and cause nuclear pathoglogy [10, 31], the mHTT- $\Delta$ N17-exon1 zebrafish represent a feasible model of HD. Thus, better understanding how N17 function is compromised in the context of HD neurons or how to improve the ability of the nucleus to clear such aberrant polyQ proteins may be important steps in reducing disease burden in HD.

Finally, our study may have important implications in the use of zebrafish models to study pathogenesis or testing candidate therapeutics for HD. An important advance we have made, through the use of conditional genetics in zebrafish, is the creation of a stable and robust model of HD with progressive movement deficits and brain pathology. The inducibility of the model allows us to study the toxicity of mutant polyQ protein in distinct cell populations. Since the model recapitulates aspects of the disease related to nuclear accumulation of mHTT-polyQ fragments, our model may be particularly useful to study mechanism and therapeutics aiming to improve nuclear proteostasis or reduce the consequence of nuclear mHTT toxcitiy in vivo. Moreover, due to the robust brain atrophy associated with the overt and progressive movement disorder, our model could also be used to test novel neuroprotective therapies. Given the obvious advantage of the zebrafish system in terms of its scalability and relative low cost in its maintenance, we envision that the mHTT- $\Delta$ N17-exon1 fish model will facilitate the screening of potential genetic disease modifiers or candidate therapeutics at a scale that is not likely to be achievable in the other vertebrate models of HD.

\section{Methods}

Animal husbandry

All zebrafish use was approved by the University of California, Los Angeles Animal Care and Use Committee. 
This work was performed under IACUC protocol ARC \# 2001-074-41 issued by OFFICE OF ANIMAL RESEARCH OVERSIGHT, University of California Los Angeles. Adults, juveniles, and zebrafish embryos were maintained according to standard zebrafish methods [64]. Heat shock was performed on shield stage embryos for $30 \mathrm{~min}$ at $38.5^{\circ} \mathrm{C}$ after which embryos were transferred to a standard zebrafish incubator at $28.5^{\circ} \mathrm{C}$ to be raised.

\section{Transgene constructs and generation of transgenic zebrafish} All transgenes were generated using the Multisite Gateway System (Life Technologies) and the Tol2kit developed for zebrafish [42]. p3E-HTT Exon 1 25Q-EGFP, p3E-HTT Exon 1 97Q-EGFP, p3E- $\Delta$ N17 HTT Exon 1 25Q-EGFP, and $\mathrm{p} 3 \mathrm{E}-\triangle \mathrm{N} 17$ HTT Exon 1 97Q-EGFP were generated by $\mathrm{BP}$ reaction of each respective $\mathrm{PCR}$ product flanked with B2F and B3R sequences to pDONR ${ }^{\mathrm{Tm}} \mathrm{P} 2 \mathrm{R}-\mathrm{P} 3$. Transgene plasmids were created by LR multisite reaction of pDESTTol2pA, p5E-bactin2, pME-loxPmCherryloxP, and each respective HTT p3E vector. Resulting plasmids were injected into single cell zebrafish embryos along with Tol2 mRNA using standard methods to efficiently generate germline integrations. Resulting $\mathrm{F}_{0}$ fish were raised and their offspring screened for ubiquitous mCherry expression. Positive $F_{1}$ embryos were raised to establish each line. Each line was derived from separate $\mathrm{F}_{0}$ fish to control for integration effects.

Cre driver lines were generated by LR multisite reaction with pDESTTol2pA, promoter specific p5E vector, pMECre, and p3E-pA. p5E-elav $3 l$ was generated by cloning the 3.1 kilobase zebrafish elav3l (also known as $\mathrm{HuC}$ ) promoter, into $\mathrm{pDONR}{ }^{\mathrm{TM}} \mathrm{P} 4 \mathrm{R}-\mathrm{P} 1 \mathrm{R}$ using the BP reaction. Similarly p5E-gfap was generated by subcloning the gfap promoter from the $\operatorname{Tg}(g f a p: G F P)$ plasmid [54] into pDONR $^{\mathrm{m}}$ P4R-P1R. p5E-mylpfa [65] and p5E-etv2 [56] were previously reported. Each transgenic line was generated as described above. However since these lines do not have a fluorescent marker gene, founders were identified by outcross to ubi:Switch fish [66] which exhibit a GFP to mCherry fluorescent switch in tissues expressing Cre recombinase. Independent lines with strong tissue specific recombination and minimal leakiness were maintained for each promoter.

\section{qRT-PCR}

Quantitative RT-PCR was performed on cDNA generated using Superscript III Reverse Transcriptase (Life Technologies, 18080044) from total RNA extracted from 5 day post fertilization larvae using Trizol reagent (Life Technologies, 15596026) on a Stratagene Mx3005P qPCR system. Three pools of ten embryos from separate clutches of each transgenic line were analyzed. Oligo(dT)12-18 (Life Technologies, 18418-012) was used to prime the reverse transcriptase reaction.
Primers were: $\beta$ actin1F, TGTTTTCCCCTCCATTGTTG; ßactin1R, ACATACATGGCAGGGGTGTT; EGFPqF, AC GTAAACGGCCACAAGTTC; EGFPqR, AAGTCGTGCT GCTTCATGTG.

\section{Western blotting}

Western blots were performed on protein extracted from 5 day post fertilization embryos using standard methods. Five embryos were pooled for each sample into $25 \mu$ of $2 \mathrm{X}$ SDS Loading Buffer. Protein samples were run on a $12 \%$ Tris-glycine acrylamide gel (Thermo Scientific, 25247). Protein was transferred onto a PDVF membrane (Thermo Scientific, 88520), and then probed with rabbit anti-GFP primary antibody 1:2000 dilution (Life Technologies, A11122), stripped and then reprobed with mouse anti-tubulin antibody 1:10,000 dilution (Sigma, T5168). Secondary antibodies were anti-rabbitperoxidase (Sigma, A0545) and anti-mouse-peroxidase (Sigma, A9044) both at 1:5000 dilutions. Chemiluminescent detection was performed using Lumi-Light western blotting reagent (Roche, 12015200001) and HyBlot CL autoradiography film (Denville Scientific, E3018). Film was digitized on an HP Scanjet G3110 and the image adjusted in Adobe Photoshop CS4. ImageJ was used to perform densitometric measurements.

\section{Behavioral analysis}

Fish were raised at similar densities and monitored weekly for abnormal movement. Fish were grouped into four categories: 1) Healthy - normal swimming; 2) Stage 1 - abnormal swimming, immobility alternating with jerky movements; 3) Stage 2 - loss of lateral stability, corkscrew swimming; 4) Stage 3 - loss of vertical stability, inability to coordinate movement, death. When fish were no longer able to swim for feeding they were killed for further analysis.

For more detailed behavioral analysis we performed electric field potential recordings on freely behaving adult elav3l:cre/HTT- $\Delta$ N17 or, Stage 1 categorized, elav3l:cre/mHTT- $\Delta \mathrm{N} 17$ fish $(n=6$ each) according to the methods of Issa et.al. [57]. Fish were placed into a recording chamber and allowed to acclimate to the chamber for $5 \mathrm{~min}$. Then field potentials generated from muscles during movement were recorded for $5 \mathrm{~min}$ for each fish. The recording chamber was $11 \mathrm{~cm}$ long $\times$ $4 \mathrm{~cm}$ wide $\times 3 \mathrm{~cm}$ deep containing double distilled water with a resistance of $\sim 15 \mathrm{M} \Omega$. Water depth was $2.75 \mathrm{~cm}$. Room temperature was set to $25^{\circ} \mathrm{C}$. Electric field potentials were recorded using one pair of electrodes $(0.5 \mathrm{~cm}$ exposure, $0.1 \mathrm{~cm}$ thick of bare copper medal) placed on either end of each testing chamber. Electric signals were amplified 1000-fold using an AC differential amplifier (AM-Systems model 1700, Carlsborg, WA USA), lowpass filtered at $300 \mathrm{~Hz}$ and high-pass filtered at $500 \mathrm{~Hz}$. 
All signals were digitized and stored with a Digidata1322A and acquired using Axoscope software (Molecular Devices, Inc., Sunnyvale, CA, USA). Data was annotated manually and movement frequency, duration, and total time were calculated.

Body length, weight, and brain weight measurements Body length and weight were measured on fish deeply anesthetized with Tricaine solution (Sigma, A5040). Length was measured from the tip of the jaw to the base of the tail since the HS:cre line was in a long-fin genetic background resulting in mixed normal and long-fin offspring. After measuring the body, fish were killed by exsanguination, the top of the skull removed, and the head fixed in $4 \%$ paraformaldehyde overnight at $4{ }^{\circ} \mathrm{C}$ to improve tissue stability for dissection. Fish heads were washed three times in PBS and gently dried. Brains were carefully dissected using watchmakers forceps from the olfactory bulb-telencephalon junction to the hindbrain-spinal cord junction. All cranial nerves were carefully removed except for the optic nerve and optic tract which remained attached to the brain. The eyes were removed. Excess liquid was removed and brain weight was measured.

\section{Histology and immunostaining}

To harvest tissue for cryo-sectioning, adult fish were deeply anesthetized in Tricaine solution (Sigma, A5040) and then killed by exsanguination. The head was removed as was the top of the skull. Fish heads were fixed overnight in $4 \%$ paraformaldahyde/PBS solution at $4{ }^{\circ} \mathrm{C}$ with constant movement on a nutator mixer. The following day the brain was dissected out intact from the tip of the forebrain to the hindbrain spinal cord junction. The brain was then fixed overnight in $4 \%$ paraformaldahyde/PBS solution plus $5 \%$ sucrose at $4{ }^{\circ} \mathrm{C}$ with constant movement on a nutator mixer. The following day fixed tissue was put through an increasing sucrose gradient: 5 , $10,12.5,15$, and $20 \%$ in PB buffer, $1 \mathrm{~h}$ each and then placed at $4{ }^{\circ} \mathrm{C}$ with constant movement on a nutator mixer overnight. The following day tissue was infiltrated with a mix of 2:1 $20 \%$ sucrose solution to OCT Compound (Tissue-Tek, 4583) for $1 \mathrm{~h}$ and then snap frozen in $100 \%$ OCT in aluminum foil molds placed into a 2-methylbutane (Sigma, M32631), dry ice bath. Blocks were stored at $-80{ }^{\circ} \mathrm{C}$ until they were cut at $16 \mu \mathrm{m}$ for immunostaining on a Leica model CM3050S cryostat.

Immunohistochemistry was performed on cryosections using the polyclonal sheep anti-human HTT primary antibody S830 [46] at 1:1000 dilution. Biotinylated anti-sheep secondary (Vector Laboratories, BA-6000) was used at 1:200 dilution and the Vectastain $A B C$ Elite kit (Vector Laboratories, PK-6100) plus Vector SG substrate (Vector Laboratories, SK-4700) used to perform the color reaction. Sections were then counter stained with Nuclear Red (Vector Laboratories, H-3403) and mounted with Permount (Fisher, SP15-100).

Immunofluorescence was performed on sections using manufacturer's recommended protocol and dilution for each antibody. Primary antibodies were rabbit anti-GFP (Life Technologies, A11122), 1/200 dilution, and anti$\mathrm{HuC} / \mathrm{HuD}$ (Life Technologies, A21271), $5 \mu \mathrm{g} / \mathrm{mL}$. Secondary antibodies were goat anti-rabbit Alexa488 IgG (Life Technologies, A11008), 1/200 dilution, and donkey anti-mouse Rhodamine (Life Technologies, A16016), 1/200 dilution. Stained sections were mounted in Prolong Gold Antifade with DAPI (Life Technologies, P36935).

\section{Imaging}

Images were captured on an Axioskop 2 plus microscope (Zeiss) using $5 \times, 10 \times$, or $40 \times$ objectives with a Hamamatsu ORCA-ER camera and Volocity 5 software (Improvision). Confocal images were collected on a Zeiss LSM 510 with a $63 \times$ oil immersion objective (NA 1.4). Adobe Photoshop CS4 was used to adjust brightness and contrast and assemble composite images.

To quantify $\mathrm{GFP}^{+}$protein aggregates in $\mathrm{HuC}$ stained sections, ImageJ was used to count cell number (DAPI positive nuclei), $\mathrm{HuC}^{+}$cells, and aggregate number. Maximum intensity projections of $133.6 \mu \mathrm{m} \times 133.6 \mu \mathrm{m} \times 4.7 \mu \mathrm{m} \mathrm{z}$ stacks were analyzed from three non-consecutive sections of each fish. Images were acquired such that bright GFP+ aggregates did not over saturate the image. Therefore, the background level of GFP expression appears lower than it actually was. Three fish were examined for each genotype.

\section{Statistical analsysis}

Student's $t$-test was used for single comparisons, ANOVA with Bonferonni posthoc test was used for multiple comparisons, and Kaplan Meier survival analysis with Log Rank posthoc test was used for survival curve comparisons with $p<0.05$ set as significant for each. All statistical analysis was performed using SPSS 14.0 software.

\section{Conclusions}

We have developed a Cre-inducible HD model in zebrafish base upon expression of human exon 1 of $H T T$. Using this model we have demonstrated that the N17 domain is protective in the context of $97 \mathrm{Q}$ since its deletion results in more severe phenotypes and earlier lethality. Deletion of N17 results in neuron-specific accumulation of 97Q in the nucleus and neuron specific induction of the transgene is sufficient to generate HD-like phenotypes. Together these results support a protective role of the $\mathrm{N} 17$ domain in $\mathrm{HD}$ possibly through nuclear export of the pathological 97Q fragment. This new zebrafish model will facilitate pharmacological, genetic, and mechanistic studies of HD. 


\section{Additional files}

Additional file 1: Movie S1. HTT- $\Delta$ N17-exon1 fish exhibiting normal swimming behavior. (MOV $19875 \mathrm{~kb}$ )

Additional file 2: Movie S2. A group of HTT- $\Delta$ N17-exon1 fish exhibiting normal swimming behavior. (MOV $6872 \mathrm{~kb}$ )

Additional file 3: Movie S3. A mHTT- $\Delta \mathrm{N} 17-$-exon1 fish exhibiting abnormal movements corresponding to Stage 2. (MOV $11321 \mathrm{~kb}$ )

Additional file 4: Movie S4. A group of $\mathrm{mHTT}-\Delta \mathrm{N} 17-\mathrm{exon} 1 \mathrm{fish}$ exhibiting abnormal movements corresponding to Stage 2 and 3. (MOV 3295 kb)

Additional file 5: $\mathrm{mHTT}-\Delta \mathrm{N} 17-e x o n 1 \mathrm{~L} 2$ develops progressive motor behavioral deficits. Similar to Line 1, Line 2 exhibits progressive movement problems corresponding to the described three stages of behavioral abnormality, resulting in immobility and death $(n=24)$. (JPEG $238 \mathrm{~kb}$ )

Additional file 6: Ubiquitous HTT-exon1-GFP expression in HTT-exon1 and $\mathbf{H T T}-\Delta \mathrm{N} 17-\mathrm{exon} 1$ lines. $3 \mathrm{D}$ projections of confocal images of brain sections from HTT-exon1 L1 (A-C) and HTT- $\Delta$ N17-exon1 L1 (D-F) stained for GFP and HuC. Note the faint nuclear GFP staining in HTT while HTT- $\Delta$ N17-exon1 has brighter nuclear GFP. (G) Relative nuclear GFP intensity measurements in $\mathrm{HuC}^{+}$cells comparing HTT-exon1-GFP versus HTT- $\Delta$ N17-exon $1, n=10$ cells each, ${ }^{* *} p<0.01$, students $t$-test. Measurments were made using ImageJ and normalized to average GFP intensity across the entire area. HTT-exon 1-GFP was arbitrarily set at $100 \%$. HTT- $\Delta \mathrm{N} 17-\mathrm{exon} 1$ nuclei were approximately $20 \%$ brighter. (JPEG 1556 kb)

Additional file 7: Quantification of cell and HTT aggregate density in $\mathrm{mHTT}$ and $\mathrm{mHTT}-\Delta \mathrm{N} 17$ fish brain sections. (A) Cell density as measured by DAPI positive nuclei per brain volume was not significantly different between the lines. (B) Percent of HuC positive cells over total DAPI positive nuclei is not different between the two lines. (C) GFP+ HTT-exon1 aggregate density is not different between the two lines. All comparisons using Student's $t$-test, $p<0.05$, not significant (ns). Cells and aggregates were quantified as described in Fig. 4 and Methods. (JPEG 223 kb)

\section{Competing interests}

The authors declare no competing interest.

\section{Authors' contributions}

MBV, YRG, XWY, and ShL conceived of the experiments. MBV and YRG performed all experiments. XG provided HTT-exon1 and HTT- $\Delta$ N17-exon1 DNA for generating the transgenes. $\mathrm{XL}$ assisted with histology and consulted on statistical analysis. WQ and SoL assisted with behavior test. MBV, XWY, and ShL wrote the manuscript with input from all authors. All authors read and approved the final manuscript.

\section{Acknowledgements}

The authors would like to thank Jason Ear for pME-LoxPmCherryLoxP, pME-Cre, and pCSDEST2-Cre, Prof. Pamela Raymond for Tg(gfap:GFP) plasmid, and Ren Xi for the HS:cre transgenic line. Work in X.W.Y.'s lab is supported by the US National Institutes of Health (NIH) NINDS grants R01 NS049501 and R01 NS074312, the Hereditary Disease Foundation, CHDI Foundation, Inc., Neuroscience of Brain Disorders Award from The McKnight Foundation and the David Weil Fund to the Semel Institute at University of California, Los Angeles (UCLA). This work was also supported by funding from Science and Technology Program of Shenzhen (CXZZ20140903101756864) and fellowship to WQ from China Postdoctoral Science Foundation.

\section{Author details}

'Department of Molecular, Cell and Developmental Biology, University of California-Los Angeles, 621 Charles E. Young Drive South, Los Angeles, CA 90095, USA. ${ }^{2}$ Center for Neurobehavioral Genetics, Semel Institute for Neuroscience and Human Behavior, Los Angeles, USA. ${ }^{3}$ Department of Psychiatry and Biobehavioral Sciences, David Geffen School of Medicine, Los Angeles, USA. ${ }^{4}$ Brain Research Institute, University of California, Los Angeles, Los Angeles, CA 90095, USA. 'Laboratory of Chemical Genomics, School of Chemical Biology and Biotechnology, Peking University Shenzhen Graduate School, Shenzhen 518055, China.
Received: 18 March 2015 Accepted: 30 November 2015

Published online: 09 December 2015

\section{References}

1. Shannon KM. Huntington's disease - clinical signs, symptoms, presymptomatic diagnosis, and diagnosis. Handb Clin Neurol. 2011;100:3-13.

2. Ross CA, Aylward EH, Wild EJ, Langbehn DR, Long JD, Warner JH, et al. Huntington disease: natural history, biomarkers and prospects for therapeutics. Nat Rev Neurol. 2014;10:204-16.

3. Walker FO. Huntington's disease. Lancet. 2007;369:218-28.

4. Orr HT, Zoghbi HY. Trinucleotide repeat disorders. Annu Rev Neurosci. 2007;30:575-621.

5. Vonsattel JP, Keller C, Cortes Ramirez EP. Huntington's disease - neuropathology. Handb Clin Neurol. 2011;100:83-100.

6. A novel gene containing a trinucleotide repeat that is expanded and unstable on Huntington's disease chromosomes. The Huntington's Disease Collaborative Research Group. Cell 1993, 72:971-983.

7. Duyao MP, Auerbach AB, Ryan A, Persichetti F, Barnes GT, McNeil SM, et al. Inactivation of the mouse Huntington's disease gene homolog $\mathrm{Hdh}$. Science. 1995;269:407-10.

8. Zeitlin S, Liu JP, Chapman DL, Papaioannou VE, Efstratiadis A. Increased apoptosis and early embryonic lethality in mice nullizygous for the Huntington's disease gene homologue. Nat Genet. 1995;11:155-63.

9. Nasir J, Floresco SB, O'Kusky JR, Diewert VM, Richman JM, Zeisler J, et al. Targeted disruption of the Huntington's disease gene results in embryonic lethality and behavioral and morphological changes in heterozygotes. Cell. 1995;81:811-23.

10. DiFiglia M, Sapp E, Chase KO, Davies SW, Bates GP, Vonsattel JP, et al. Aggregation of huntingtin in neuronal intranuclear inclusions and dystrophic neurites in brain. Science. 1997;277:1990-3.

11. Davies SW, Turmaine M, Cozens BA, DiFiglia M, Sharp AH, Ross CA, et al. Formation of neuronal intranuclear inclusions underlies the neurological dysfunction in mice transgenic for the HD mutation. Cell. 1997;90:537-48.

12. Gutekunst CA, Li SH, Yi H, Mulroy JS, Kuemmerle S, Jones R, et al. Nuclear and neuropil aggregates in Huntington's disease: relationship to neuropathology. J Neurosci. 1999;19:2522-34.

13. Arrasate M, Mitra S, Schweitzer ES, Segal MR, Finkbeiner S. Inclusion body formation reduces levels of mutant huntingtin and the risk of neuronal death. Nature. 2004;431:805-10.

14. Peters MF, Nucifora FC, Kushi J, Seaman HC, Cooper JK, Herring WJ, et al. Nuclear targeting of mutant Huntingtin increases toxicity. Mol Cell Neurosci. 1999;14:121-8.

15. Schilling G, Savonenko AV, Klevytska A, Morton JL, Tucker SM, Poirier M, et al. Nuclear-targeting of mutant huntingtin fragments produces Huntington's disease-like phenotypes in transgenic mice. Hum Mol Genet. 2004;13:1599-610.

16. Truant R, Atwal RS, Burtnik A. Nucleocytoplasmic trafficking and transcription effects of huntingtin in Huntington's disease. Prog Neurobiol. 2007;83:211-27.

17. Steffan JS, Agrawal N, Pallos J, Rockabrand E, Trotman LC, Slepko N, et al. SUMO modification of Huntingtin and Huntington's disease pathology. Science. 2004;304:100-4.

18. Tartari M, Gissi C, Lo Sardo V, Zuccato C, Picardi E, Pesole G, et al. Phylogenetic comparison of huntingtin homologues reveals the appearance of a primitive polyQ in sea urchin. Mol Biol Evol. 2008;25:330-8.

19. Atwal RS, Xia J, Pinchev D, Taylor J, Epand RM, Truant R. Huntingtin has a membrane association signal that can modulate huntingtin aggregation, nuclear entry and toxicity. Hum Mol Genet. 2007;16:2600-15.

20. Rockabrand E, Slepko N, Pantalone A, Nukala VN, Kazantsev A, Marsh JL, et al. The first 17 amino acids of Huntingtin modulate its sub-cellular localization, aggregation and effects on calcium homeostasis. Hum Mol Genet. 2007;16:61-77.

21. Kim MW, Chelliah Y, Kim SW, Otwinowski Z, Bezprozvanny I. Secondary structure of Huntingtin amino-terminal region. Structure. 2009;17:1205-12.

22. Michalek M, Salnikov ES, Werten S, Bechinger B. Membrane interactions of the amphipathic amino terminus of huntingtin. Biochemistry. 2013;52:847-58.

23. Thakur AK, Jayaraman M, Mishra R, Thakur M, Chellgren VM, Byeon IJ, et al. Polyglutamine disruption of the huntingtin exon $1 \mathrm{~N}$ terminus triggers a complex aggregation mechanism. Nat Struct Mol Biol. 2009; 16:380-9. 
24. Tam S, Spiess C, Auyeung W, Joachimiak L, Chen B, Poirier MA, et al. The chaperonin TRiC blocks a huntingtin sequence element that promotes the conformational switch to aggregation. Nat Struct Mol Biol. 2009;16:1279-85.

25. Lee CY, Cantle JP, Yang XW. Genetic manipulations of mutant huntingtin in mice: new insights into Huntington's disease pathogenesis. FEBS J. 2013:280:4382-94.

26. Thompson LM, Aiken CT, Kaltenbach LS, Agrawal N, Illes K, Khoshnan A, et al. IKK phosphorylates Huntingtin and targets it for degradation by the proteasome and lysosome. J Cell Biol. 2009;187:1083-99.

27. Gu X, Greiner ER, Mishra R, Kodali R, Osmand A, Finkbeiner S, et al. Serines 13 and 16 are critical determinants of full-length human mutant huntingtin induced disease pathogenesis in HD mice. Neuron. 2009;64:828-40.

28. Gu X, Cantle JP, Greiner ER, Lee CY, Barth AM, Gao F, et al. N17 Modifies Mutant Huntingtin Nuclear Pathogenesis and Severity of Disease in HD BAC Transgenic Mice. Neuron. 2015;85:726-41.

29. Gray M, Shirasaki DI, Cepeda C, André VM, Wilburn B, Lu XH, et al. Full-length human mutant huntingtin with a stable polyglutamine repeat can elicit progressive and selective neuropathogenesis in BACHD mice. J Neurosci. 2008;28:6182-95.

30. Crook ZR, Housman D. Huntington's disease: can mice lead the way to treatment? Neuron. 2011;69:423-35.

31. Landles C, Sathasivam K, Weiss A, Woodman B, Moffitt H, Finkbeiner S, et al. Proteolysis of mutant huntingtin produces an exon 1 fragment that accumulates as an aggregated protein in neuronal nuclei in Huntington disease. J Biol Chem. 2010;285:8808-23.

32. Sathasivam K, Neueder A, Gipson TA, Landles C, Benjamin AC, Bondulich MK, et al. Aberrant splicing of HTT generates the pathogenic exon 1 protein in Huntington disease. Proc Natl Acad Sci U S A. 2013;110:2366-70.

33. Mangiarini L, Sathasivam K, Seller M, Cozens B, Harper A, Hetherington C, et al. Exon 1 of the HD gene with an expanded CAG repeat is sufficient to cause a progressive neurological phenotype in transgenic mice. Cell. 1996;87:493-506

34. Krobitsch S, Lindquist S. Aggregation of huntingtin in yeast varies with the length of the polyglutamine expansion and the expression of chaperone proteins. Proc Natl Acad Sci U S A. 2000;97:1589-94.

35. Faber PW, Alter JR, MacDonald ME, Hart AC. Polyglutamine-mediated dysfunction and apoptotic death of a Caenorhabditis elegans sensory neuron. Proc Natl Acad Sci U S A. 1999;96:179-84.

36. Parker JA, Connolly JB, Wellington C, Hayden M, Dausset J, Neri C. Expanded polyglutamines in Caenorhabditis elegans cause axonal abnormalities and severe dysfunction of PLM mechanosensory neurons without cell death. Proc Natl Acad Sci U S A. 2001;98:13318-23.

37. Jackson GR, Salecker I, Dong X, Yao X, Arnheim N, Faber PW, et al. Polyglutamine-expanded human huntingtin transgenes induce degeneration of Drosophila photoreceptor neurons. Neuron. 1998;21:633-42.

38. Xi Y, Noble S, Ekker M. Modeling neurodegeneration in zebrafish. Curr Neurol Neurosci Rep. 2011;11:274-82.

39. Schiffer NW, Broadley SA, Hirschberger T, Tavan P, Kretzschmar HA, Giese A, et al. Identification of anti-prion compounds as efficient inhibitors of polyglutamine protein aggregation in a zebrafish model. J Biol Chem. 2007;282:9195-203.

40. Miller VM, Nelson RF, Gouvion CM, Williams A, Rodriguez-Lebron E, Harper SQ, et al. CHIP suppresses polyglutamine aggregation and toxicity in vitro and in vivo. J Neurosci. 2005;25:9152-61.

41. Williams A, Sarkar S, Cuddon P, Ttofi EK, Saiki S, Siddiqi FH, et al. Novel targets for Huntington's disease in an mTOR-independent autophagy pathway. Nat Chem Biol. 2008;4:295-305.

42. Kwan KM, Fujimoto E, Grabher C, Mangum BD, Hardy ME, Campbell DS, et al. The Tol2kit: a multisite gateway-based construction kit for Tol2 transposon transgenesis constructs. Dev Dyn. 2007;236:3088-99.

43. Tsai SB, Tucci V, Uchiyama J, Fabian NJ, Lin MC, Bayliss PE, et al. Differential effects of genotoxic stress on both concurrent body growth and gradual senescence in the adult zebrafish. Aging Cell. 2007;6:209-24.

44. Zeng L, Tallaksen-Greene SJ, Wang B, Albin RL, Paulson HL. The de-ubiquitinating enzyme ataxin-3 does not modulate disease progression in a knock-in mouse model of Huntington disease. J Huntingtons Dis. 2013;2:201-15.

45. Maiuri T, Woloshansky T, Xia J, Truant R. The huntingtin N17 domain is a multifunctional CRM1 and Ran-dependent nuclear and cilial export signal. Hum Mol Genet. 2013;22:1383-94.
46. Sathasivam K, Woodman B, Mahal A, Bertaux F, Wanker EE, Shima DT, et al. Centrosome disorganization in fibroblast cultures derived from R6/2 Huntington's disease (HD) transgenic mice and HD patients. Hum Mol Genet. 2001;10:2425-35.

47. Hsiao HY, Chern Y. Targeting glial cells to elucidate the pathogenesis of Huntington's disease. Mol Neurobiol. 2010;41:248-55.

48. Crotti A, Benner C, Kerman BE, Gosselin D, Lagier-Tourenne C, Zuccato C, et al. Mutant Huntingtin promotes autonomous microglia activation via myeloid lineage-determining factors. Nat Neurosci. 2014;17:513-21.

49. She P, Zhang Z, Marchionini D, Diaz WC, Jetton TJ, Kimball SR, et al. Molecular characterization of skeletal muscle atrophy in the R6/2 mouse model of Huntington's disease. Am J Physiol Endocrinol Metab. 2011;301:E49-61.

50. Rahman A, Ekman M, Shakirova Y, Andersson KE, Mörgelin M, Erjefält JS, et al. Late onset vascular dysfunction in the R6/1 model of Huntington's disease. Eur J Pharmacol. 2013;698:345-53.

51. Lin CY, Hsu YH, Lin MH, Yang TH, Chen HM, Chen YC, et al. Neurovascular abnormalities in humans and mice with Huntington's disease. Exp Neurol. 2013;250:20-30.

52. Wang N, Gray M, Lu XH, Cantle JP, Holley SM, Greiner E, et al. Neuronal targets for reducing mutant huntingtin expression to ameliorate disease in a mouse model of Huntington's disease. Nat Med. 2014;20:536-41.

53. Park HC, Kim CH, Bae YK, Yeo SY, Kim SH, Hong SK, et al. Analysis of upstream elements in the HuC promoter leads to the establishment of transgenic zebrafish with fluorescent neurons. Dev Biol. 2000;227:279-93.

54. Bernardos RL, Raymond PA. GFAP transgenic zebrafish. Gene Expr Patterns. 2006;6:1007-13.

55. Ju B, Chong SW, He J, Wang X, Xu Y, Wan H, et al. Recapitulation of fast skeletal muscle development in zebrafish by transgenic expression of GFP under the mylz2 promoter. Dev Dyn. 2003;227:14-26.

56. Veldman MB, Lin S. Etsrp/Etv2 is directly regulated by Foxc1a/b in the zebrafish angioblast. Circ Res. 2012;110:220-9.

57. Issa FA, O'Brien G, Kettunen P, Sagasti A, Glanzman DL, Papazian DM. Neural circuit activity in freely behaving zebrafish (Danio rerio). J Exp Biol. 2011;214:1028-38.

58. von Hörsten S, Schmitt I, Nguyen HP, Holzmann C, Schmidt T, Walther T, et al. Transgenic rat model of Huntington's disease. Hum Mol Genet. 2003;12:617-24.

59. Slow EJ, van Raamsdonk J, Rogers D, Coleman SH, Graham RK, Deng Y, et al. Selective striatal neuronal loss in a YAC128 mouse model of Huntington disease. Hum Mol Genet. 2003;12:1555-67.

60. Gu X, Li C, Wei W, Lo V, Gong S, Li SH, et al. Pathological cell-cell interactions elicited by a neuropathogenic form of mutant Huntingtin contribute to cortical pathogenesis in HD mice. Neuron. 2005;46:433-44.

61. Gu X, André VM, Cepeda C, Li SH, Li XJ, Levine MS, et al. Pathological cell-cell interactions are necessary for striatal pathogenesis in a conditional mouse model of Huntington's disease. Mol Neurodegener. 2007;2:8.

62. Zheng Z, Li A, Holmes BB, Marasa JC, Diamond MI. An N-terminal nuclear export signal regulates trafficking and aggregation of Huntingtin $(\mathrm{Htt})$ protein exon 1. J Biol Chem. 2013;288:6063-71.

63. Ordway JM, Tallaksen-Greene S, Gutekunst CA, Bernstein EM, Cearley JA, Wiener HW, et al. Ectopically expressed CAG repeats cause intranuclear inclusions and a progressive late onset neurological phenotype in the mouse. Cell. 1997;91:753-63.

64. Westerfield M. The zebrafish book: a guide for the laboratory use of zebrafish (Brachydanio rerio). Eugene: M. Westerfield; 1993.

65. Veldman MB, Zhao C, Gomez GA, Lindgren AG, Huang $H$, Yang $H$, et al. Transdifferentiation of fast skeletal muscle into functional endothelium in vivo by transcription factor Etv2. PLoS Biol. 2013;11:e1001590.

66. Mosimann C, Kaufman CK, Li P, Pugach EK, Tamplin OJ, Zon LI. Ubiquitous transgene expression and Cre-based recombination driven by the ubiquitin promoter in zebrafish. Development. 2011;138:169-77. 